\title{
Transient Left Ventricular Dysfunction After Percutaneous Patent Ductus Arteriosus Closure in Children
}

\author{
Yeo Hyang Kim, MD², Hee Joung Choi, MD², Yongkeun Cho, MD³ , Sang Bum Lee, MD and Myung Chul Hyun, MD \\ ${ }^{1}$ Department of Pediatrics, School of Medicine, Keimyung University, Daegu, \\ ${ }^{2}$ Department of Pediatrics, College of Medicine, Pochon CHA University, Gumi, \\ ${ }^{3}$ Department of Internal Medicine and ${ }^{4}$ Pediatrics, School of Medicine, Kyungpook National University, Daegu, Korea
}

\section{ABSTRACT}

Background and Objectives: The goal of this study was to assess changes in left ventricular (LV) function and to identify pre-closure factors associated with LV dysfunction \{fractional shortening (FS) below 29\% after transcatheter patent ductus arteriosus (PDA) closure. Subjects and Methods: Forty-three pediatric patients with PDAs underwent cardiac catheterization for hemodynamic studies and intervention. Doppler echocardiography was performed at pre-closure, post-closure, and follow-up. Results: S' and A' of the septum and mitral annulus were significantly decreased at post-closure and follow-up, respectively. In five of eight patients with Qp/Qs ratios over 1.60 and $\mathrm{Pp} / \mathrm{Ps}$ ratios over 0.32 at pre-closure, the FS was decreased below 29\% at post-closure. Qp/Qs ratio over 1.60 and $\mathrm{Pp} / \mathrm{Ps}$ ratio over 0.32 at pre-closure had a sensitivity of $86 \%$ and a specificity of $84 \%$ for predicting FS to be below 29\% at post-closure. Conclusion: Larger amounts of pre-closure left-to-right shunting and higher pulmonary artery pressure were associated with an increased likelihood of FS $\angle 29 \%$ after closure. The results of this study suggest that serial assessments of ventricular function are needed after PDA occlusion in patients with high Qp/Qs and Pp/Ps ratios. (Korean Circ J 2008;38:596-600)

KEY WORDS: Ductus arteriosus, patent; Catheter closure; Ventricular dysfunction.

\section{Introduction}

Patent ductus arteriosus (PDA) is a congenital heart disease that results in left-to-right shunting; it is associated with increased left ventricular (LV) preload. ${ }^{\left.1{ }^{12}\right)}$ Over the last 30 years, treatment of most PDAs has been accomplished by transcatheter interventional closure using various devices, such as detachable coils, Nit-Occluders, and Amplatzer duct occluders, ${ }^{3-8)}$ although video-assisted thoracoscopic surgery has been introduced for large PDAs. ${ }^{9)}$ Previous studies have reported an immediate change in LV performance followed by recovery within a few months after successful transcatheter closure of PDAs in children. ${ }^{2) 10}$ However, some cases with larger ductus size and higher pulmonary pressure had immediate LV dysfunction ffractional shortening (FS) below 29\%\} after interventional PDA closure.

\footnotetext{
Received: July 9, 2008

Revision Received: September 1, 2008

Accepted: September 17, 2008

Correspondence: Myung Chul Hyun, MD, Department of Pediatrics, Schoo of Medicine, Kyungpook National University, 200 Dongdeong-no, Junggu, Daegu 700-721, Korea

Tel: 82-53-420-5704, Fax: 82-53-425-6683

E-mail:mchyun@mail.knu.ac.kr
}

Echocardiography is the most commonly used method for evaluating LV function; such studies include 2dimensional (2-D), M-mode, conventional Doppler, and tissue Doppler technology. The purpose of this study was to investigate changes in LV function before and after percutaneous transcatheter PDA closure using conventional and tissue Doppler echocardiography (TDE). In addition, we evaluated pre-closure factors associated with immediate LV dysfunction after the procedure.

\section{Subjects and Methods}

\section{Study population}

Forty-three pediatric patients were enrolled in this study. They were all diagnosed with PDAs, and they all underwent successful percutaneous transcatheter PDA closure between January 2005 and May 2007. Patients of unsuitable size for interventional closure or with additional congenital heart defects were excluded. Echocardiography was performed to detect combined cardiac defects and to analyze ventricular function. Study approval was obtained from the institutional ethics committee at the Kyungpook National University School of Medicine, and written informed consent was obtained 
from the patients' parents in all cases.

\section{Two-dimensional and tissue Doppler echocardiog- raphy}

Echocardiography was performed with the patient in the supine position using an Acuson Sequoia (Siemens Medical Solution, Mountain View, CA, USA) with a 4 $\mathrm{MHz}$ transducer at pre-closure, post-closure (the next or second day after the procedure), and follow-up (3 months after the procedure). Some patients required sedation for echocardiography. The LV end-diastolic dimension (LVEDD) and LV end-systolic dimension (LVESD) were measured in the parasternal long-axis view. Fractional shortening (FS) was calculated as (LVEDD-LVESD)/ LVEDD $\times \times 100$. We defined FS below $29 \%$ as "Abnormal" ${ }^{\prime 1)}$ and considered FS changes 10\% greater than the pre-closure level as "Change". ${ }^{2)}$ For diastolic functional analysis, the mitral inflow signal was acquired from three cardiac cycles in the apical four-chamber view. The pulsed Doppler sample volume was $2 \mathrm{~mm}$, placed at the mitral valve tip. The early peak flow velocity $(\mathrm{E})$ and atrial filling velocity (A) were measured three times and averaged, and the E/A ratio was calculated. In addition, the peak systolic S', peak early diastolic E', and peak late diastolic A' velocities were obtained from the TDE using the pulsed wave Doppler method at the septal corner, and the lateral portion of the mitral annulus and the E'/A' and E/E' ratios were calculated. The TDE was recorded from the apical four-chamber view, and the sample volume was $2 \mathrm{~mm}$.

\section{Cardiac catheterization}

All patients received a first generation cephalosporin injection $(50 \mathrm{mg} / \mathrm{kg}) 30$ minutes before the procedure, were sedated with ketamine, midazolam, or propofol during the procedure, and also received a dose of 50$100 \mathrm{unit} / \mathrm{kg}$ of heparin after vein and artery puncture. Hemodynamic information was obtained from a standard cardiac catheterization, and the pulmonary blood flow/systemic blood flow (Qp/Qs), pulmonary artery pressure/systemic artery pressure $(\mathrm{Pp} / \mathrm{Ps})$, and pulmonary artery resistance/systemic artery resistance (Rp/Rs) ratios were calculated before PDA closure using the Fick principle. A lateral aortogram was performed at the distal aortic arch before PDA closure, followed by PDA sizing in an appropriate image view. Percutaneous transcatheter closure of the PDA was performed using antegrade or retrograde technique, according to the device type. Nearly complete or complete closure was confirmed by repeat aortogram after the procedure.

\section{Statistical analysis}

Statistical analysis was carried out using the statistical package for social science (SPSS) software program for Windows (version 12; SPSS, Chicago, Illinois, USA).
Continuous variables were compared using the Student $\mathrm{t}$-test and are expressed as mean values \pm standard deviation. Changes in echocardiographic parameters were compared using the paired t-test. Correlation between two continuous variables was assessed using linear regression analysis. Multiple stepwise linear regression analysis was used to identify pre-closure determinants of post-closure FS below $29 \%$. Univariate analysis was performed first, and a $p<0.05$ was considered statistically significant. Multivariate analysis was then performed using variables that were significant on univariate analysis; a $\mathrm{p}<0.1$ was considered significant. The best cutoff value for a FS below $29 \%$ post-closure was identified based on the receiver operating characteristic curve analysis.

\section{Results}

\section{Clinical characteristics}

The clinical characteristics of the patients are summarized in Table 1. There were 14 boys and 29 girls, with a median age of 24 months at the time of the procedure (range 6-97 months). The mean narrowest PDA diameter measured by the aortogram was $2.0 \pm 1.3 \mathrm{~mm}$ (range 1.9-5.5 mm). All patients underwent percutaneous transcatheter closure using devices such as Amplatzer duct occluders, Nit-Occluders, and detachable coils. PDA occlusions were performed through an antegrade approach using the Amplatzer duct occluder (AGA Medical Corp., Golden Valley, MN, USA) or Nit-Occluder in 26 pa- $^{-}$ tients and a retrograde approach using a detachable coil in 13 patients. Both the Nit-Occluder and the detachable coil were used in four patients.

\section{Two-dimensional and tissue Doppler echocardiog- raphy \\ Compared to pre-closure values, the FS was signif-}

Table 1. Clinical characteristics of patients

\begin{tabular}{lcc}
\hline & PDA patients $(\mathrm{n}=43)$ & Range \\
\hline Age (mo) & $24.1 \pm 19.1$ & $6-97$ \\
Weight $(\mathrm{kg})$ & $11.7 \pm 4.0$ & $5.7-17.8$ \\
BSA $\left(\mathrm{m}^{2}\right)$ & $0.4 \pm 0.1$ & $0.19-0.59$ \\
Pulmonic end size (mm) & $2.0 \pm 1.3$ & $1.9-5.9$ \\
Qp/Qs & $1.43 \pm 0.61$ & $1-3.30$ \\
Pp/Ps & $0.30 \pm 0.18$ & $0.11-0.91$ \\
Rp/Rs & $0.13 \pm 0.10$ & $0.02-0.44$ \\
Device for intervention & & \\
Amplatzer & 15 & \\
Nit occluder & 11 & \\
Detachable coil & 4 & \\
Nit occluder+detachable coil & & \\
\hline PDA: patent ductus arteriosus, BSA: body surface area, Qp/Qs: \\
pulmonary blood flow/systemic blood flow, Pp/Ps: pulmonary artery \\
pressure/systemic artery pressure, Rp/Rs: pulmonary artery resistance/ \\
systemic artery resistance
\end{tabular}


icantly decreased post-closure $(p<0.01)$, but recovered at follow-up when it returned to pre-closure values (Table 2). The LVEDD was significantly decreased postclosure $(\mathrm{p}<0.01)$ and showed a persistent decrease at follow-up. However, there was no significant difference be-

Table 2. Changes in parameters on conventional and tissue Doppler echocardiography

\begin{tabular}{lccc}
\hline & Pre-closure & Post-closure & Follow-up \\
\hline FS (\%) & $37.6 \pm 4.6$ & $33.3 \pm 5.9^{*}$ & $36.3 \pm 5.8^{\dagger}$ \\
LVEDD (cm) & $3.30 \pm 0.48$ & $3.07 \pm 0.49^{*}$ & $2.98 \pm 0.45^{*}$ \\
LVESD (cm) & $2.06 \pm 0.37$ & $2.06 \pm 0.40$ & $1.91 \pm 0.38^{\ddagger}$ \\
Septal & & & \\
S' (cm/s) & $7.86 \pm 2.15$ & $7.43 \pm 1.55^{*}$ & $7.49 \pm 1.86^{*}$ \\
E' (cm/s) & $11.85 \pm 2.79$ & $12.12 \pm 3.52$ & $11.77 \pm 2.51$ \\
A' (cm/s) & $8.46 \pm 2.95$ & $7.79 \pm 2.84$ & $7.78 \pm 2.29^{*}$ \\
E/E' & $9.11 \pm 3.00$ & $8.87 \pm 3.45$ & $8.70 \pm 2.56$ \\
Mitral lateral & & & \\
S' (cm/s) & $7.55 \pm 2.35$ & $6.72 \pm 2.01^{*}$ & $7.03 \pm 1.55$ \\
E' (cm/s) & $13.79 \pm 4.07$ & $12.79 \pm 4.40$ & $13.71 \pm 4.42$ \\
A' (cm/s) & $8.59 \pm 3.72$ & $7.54 \pm 2.95$ & $7.10 \pm 2.30^{*}$ \\
E/E' & $8.63 \pm 3.27$ & $9.36 \pm 5.74$ & $7.50 \pm 2.16$ \\
\hline
\end{tabular}

${ }^{*} \mathrm{p}<0.01$ versus pre-closure, ${ }^{\dagger} \mathrm{p}<0.01$ versus post-closure, ${ }^{\ddagger} \mathrm{p}<0.01$ versus pre- and post-closure. FS: fractional shortening, LVEDD: left ventricular end-diastolic dimension, LVESD: left ventricular endsystolic dimension. S': peak systolic velocity in tissue Doppler imaging, E': peak early diastolic velocity in tissue Doppler imaging, A': peak late diastolic velocity in tissue Doppler imaging

Table 3. Multiple linear regression analysis for FS below $29 \%$ at post-closure

\begin{tabular}{lcc}
\hline Pre-closure variables & Univariate P & Multivariate P \\
\hline FS (\%) & 0.544 & \\
Age $(\mathrm{mo})$ & 0.278 & \\
Wt $(\mathrm{kg})$ & 0.297 & \\
BSA $\left(\mathrm{m}^{2}\right)$ & 0.283 & \\
Pulmonic end size (mm) & 0.000 & 0.077 \\
Qp/Qs & 0.000 & 0.030 \\
Pp/Ps & 0.000 & \\
Rp/Rs & 0.003 & \\
LVEDD (mm) & 0.046 & \\
LVESD (mm) & 0.035 & \\
LAD (mm) & 0.348 & \\
\hline
\end{tabular}

FS: fractional shortening, Wt: weight, BSA: body surface area, LVEDD: left ventricular end-diastolic dimension, LVESD: left ventricular end-systolic dimension, LAD: left atrial dimension, Qp/Qs: pulmonary blood flow/systemic blood flow, Pp/Ps: pulmonary artery pressure/systemic artery pressure, Rp/Rs: pulmonary artery resistance/ systemic artery resistance tween the post-closure and follow-up values. The LVESD was no different in a comparison of pre- and post-closure values, despite the significant decrease at followup. There was no significant interval change in the E, $A$, or $E / A$ ratio based on the mitral inflow signal $(p>$ 0.1 ). The TDE of the septum and mitral lateral annulus showed that the S' was significantly decreased post-closure and the $A^{\prime}$ was significantly decreased at follow-up (Table 2). The S' values at post-closure and follow-up, the E' values at all examination times, and the A'values at pre- and post-closure were all similar $(\mathrm{p}>0.05)$.

\section{Parameters associated with left ventricular dysfunction}

In order to identify the factors associated with a postclosure decrease in the FS below 29\%, stepwise multiple linear regression analysis was performed (Table 3). Univariate stepwise linear regression analysis showed that the LVEDD and the pulmonic end size obtained from the lateral aortogram, $\mathrm{Qp} / \mathrm{Q}, \mathrm{Pp} / \mathrm{Ps}$, and $\mathrm{Rp} / \mathrm{Rs}$ ratios were associated with a FS below $29 \%$ at post-closure examination $(\mathrm{p}<0.05)$. Among these parameters, the Qp/ $\mathrm{Qs}$ and $\mathrm{Pp} / \mathrm{Ps}$ ratios were statistically significant factors on multivariate stepwise linear regression analysis for post-closure FS below 29\% $(\mathrm{p}<0.1)$. The receiver operating characteristic curve analysis showed that a $\mathrm{Qp} /$ Qs ratio over 1.60 and a $\mathrm{Pp} / \mathrm{Ps}$ ratio over 0.32 were the best cutoff values for post-closure FS below 29\%, with a sensitivity of $86 \%$ and a specificity of $84 \%$.

Table 4 lists the post-closure FS values, FS changes between pre- and post-closure, and Qp/Qs and $\mathrm{Pp} / \mathrm{Ps}$ ratios. Changes in FS between pre- and post-closure were below 10\%, 10-20\%, and over 20\% in 21 patients, 8 patients, and 14 patients, respectively. Changes in FS of over $10 \%$ were considered to be a "Change", and 22 patients had such changes. A significant "Change" in FS was noted in $40 \%(14 / 35)$ of patients with postclosure FS equal to or greater than $29 \%$ and in $100 \%$ (8/8) of patients with post-closure FS below 29\%. The Qp/Qs ratio was over 1.60 in 12 of 43 patients and below 1.60 in 31 patients. Fifty-eight percent $(7 / 12)$ of patients with a $\mathrm{Qp} / \mathrm{Q}$ s ratio over $1.60 \mathrm{had}$ a post-closure FS below 29\% compared with 3\% (1/31) of patients with a $\mathrm{Qp} / \mathrm{Q}$ s ratio below 1.60. In addition, the $\mathrm{Pp} / \mathrm{Ps}$ ratio was over 0.32 in 12 of 43 patients and below 0.32 in 31 patients. Among patients with a $\mathrm{Pp} / \mathrm{Ps}$ ratio over 0.32, the post-closure FS was below $29 \%$ in $50 \%(6 / 12)$

Table 4. Patients according to post-closure changes in FS, Qp/Qs, and Pp/Ps

\begin{tabular}{|c|c|c|c|c|c|c|c|c|}
\hline & & \multicolumn{3}{|c|}{ FS change } & \multicolumn{2}{|c|}{$\mathrm{Qp} / \mathrm{Qs}$} & \multicolumn{2}{|c|}{$\mathrm{Pp} / \mathrm{Ps}$} \\
\hline & & $<10 \%$ & $10-20 \%$ & $>20 \%$ & $>1.60$ & $\leq 1.60$ & $>0.32$ & $\leq 0.32$ \\
\hline \multirow{2}{*}{ Post-closure FS } & $>29 \%$ & 21 & 7 & 7 & 5 & 30 & 6 & 29 \\
\hline & $\leq 29 \%$ & 0 & 1 & 7 & 7 & 1 & 6 & 2 \\
\hline
\end{tabular}

FS: fractional shortening, Qp/Qs: pulmonary blood flow/systemic blood flow, Pp/Ps: pulmonary artery pressure/systemic artery pressure, Rp/Rs: pulmonary artery resistance/systemic artery resistance 


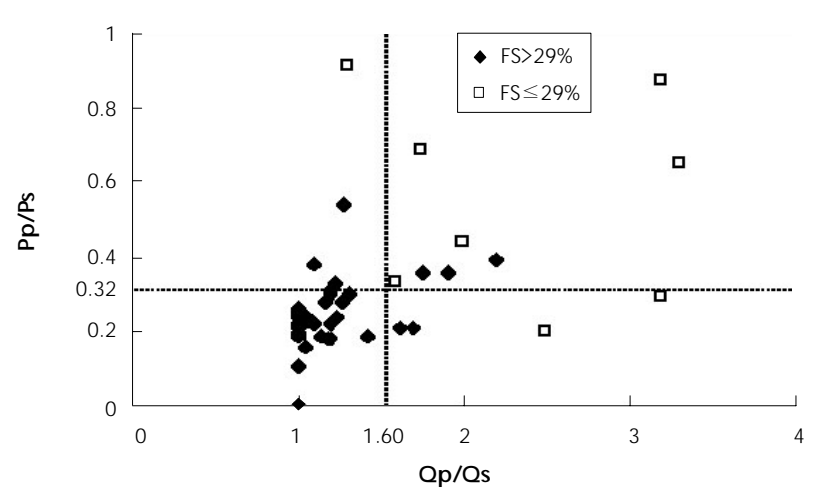

Fig. 1. Distribution of each patient according to $\mathrm{Qp} / \mathrm{Qs}$ and $\mathrm{Pp} /$ Ps. Qp/Qs $=1.60$ and $P p / P s=0.32$ were the best cutoff values for post-closure $\mathrm{FS}$ below $29 \%$, and these figures yielded a sensitivity of $86 \%$ and a specificity of $84 \%$. More patients showed LV "dysfunction" with a Qp/Qs ratio over 1.60 and a Pp/Ps ratio over 0.32 , compared to the others. FS: fractional shortening, LV: left ventricle, Qp/Qs: pulmonary blood flow/systemic blood flow, Pp/Ps: pulmonary artery pressure/systemic artery pressure, Rp/Rs: pulmonary artery resistance/systemic artery resistance.

of them, compared with $6 \%(2 / 31)$ of the patients with a Qp/Qs ratio below 1.60.

Eight patients had a Qp/Qs ratio over 1.60 and a $\mathrm{Pp} /$ Ps ratio over 0.32 (Fig. 1). Five of eight patients had LV "dysfunction" with post-closure FS below 29\% and FS change of over 20\%. Post-closure FS below 29\% was observed in one patient with a Qp/Qs ratio below 1.60 and a $\mathrm{Pp} / \mathrm{Ps}$ ratio over 0.32 , and in two patients with $\mathrm{Qp} / \mathrm{Qs}$ ratios over 1.60 and $\mathrm{Pp} / \mathrm{Ps}$ ratios below 0.32 . No patients with $\mathrm{Qp} / \mathrm{Qs}$ ratios below 1.60 and $\mathrm{Pp} / \mathrm{Ps}$ ratios below 0.32 had post-closure FS below $29 \%$.

\section{Discussion}

Transcatheter interventional closure of PDAs has been practiced with various devices since $1967 .{ }^{12)} \mathrm{Re}-$ cently, with the introduction of the Amplatzer ductal occluder into clinical practice, transcatheter closure has become possible for even very large PDAs. ${ }^{13)}$

Because a PDA is a congenital heart defect with a left-to-right shunt, it is associated with an increased LV preload. PDA closure causes an immediate reduction in ventricular preload. ${ }^{12)(4)}$ Prior reports ${ }^{15)}$ have shown that patients with PDAs differ from normal control subjects in LV volume and function as measured by twoand three-dimensional echocardiography at baseline. These changes caused by the PDA generally resolve by six months after percutaneous closure. Galal et al. ${ }^{2)}$ demonstrated that patients with large PDAs (larger than 3.1 $\mathrm{mm}$ ) had significant deterioration in FS immediately after PDA closure, which was followed by FS recovery within six months.

Compared to the values before PDA closure, in this study, a significant decrease in the LVEDD was noted; there was no difference in the LVESD immediately after PDA closure. However, at follow-up, an additional decrease in the LVEDD and a significant decrease in the LVESD were detected. An immediate decrease in the LVEDD and a late decrease in the LVESD resulted in a change in the FS when pre-closure, post-closure, and follow-up values were compared. Although FS is load-dependent and an acute change in LV volume loading after PDA closure is the primary underlying cause of this change in FS; FS below 29\% after PDA closure was unusual and was considered to reflect LV dysfunction.

This study showed that LV dysfunction was present in eight patients immediately after PDA closure. Among these eight patients with LV dysfunction, seven patients had FS changes over 20\%, five had Qp/Qs ratios over 1.60 and $\mathrm{Pp} / \mathrm{Ps}$ ratios over 0.32 , and two patients each had only Qp/Qs ratios over 1.60 or $\mathrm{Pp} / \mathrm{Ps}$ ratios over 0.32 . All of the patients were medicated with digitalis or angiotensin converting enzyme (ACE) inhibitors, but none showed clinical symptoms of ventricular dysfunction. In one case with a post-closure FS below 29\% and a FS change below $20 \%$, the FS was $28 \%$ pre-closure, $23 \%$ post-closure, and $25 \%$ at follow-up, and the Qp/ Qs and $\mathrm{Pp} / \mathrm{Ps}$ ratios were 1.60 and 0.33 , respectively. This patient may have had a cardiomyopathy concurrent with the PDA. However, further diagnostic evaluations such as a cardiac biopsy were not performed, and the patient has been undergoing regular follow-up.

The relationship of the $\mathrm{Qp} / \mathrm{Qs}$ and $\mathrm{Pp} / \mathrm{Ps}$ ratios with FS was thought to be explained by larger amounts of shunting, higher pulmonary artery pressure, greater changes in the FS, and significant LV dysfunction. Therefore, based on our findings, although the patients had normal ventricular function before the procedure, patients with $\mathrm{Qp} / \mathrm{Qs}$ ratios over 1.60 and $\mathrm{Pp} / \mathrm{Ps}$ ratios over 0.32 at catheterization are predicted to be at risk for deterioration of ventricular function after the procedure. These patients require early evaluation of ventricular function using echocardiography.

Jeong et al. ${ }^{16)}$ reported persistent LV systolic dysfunction $(\mathrm{EF}<50 \%)$ after PDA closure in adult PDA patients with low pre-closure LV EF. In contrast to Jeong's study, our study showed recovery of the FS at follow-up, despite the immediate post-closure LV systolic dysfunction (FS<29\%). This discrepancy might be due to differences in the populations studied (adults vs. children). A longer duration of LV volume overload may cause changes in LV contractility and may lead to incomplete recovery after reduction in the loading condition. The ventricular remodeling status is different according to patient age. In this study, the tissue Doppler myocardial velocity was significantly decreased during the systolic and diastolic phases at post-closure and follow-up compared to the pre-closure measurements. These findings suggest that an immediate reduction in the preload after PDA closure may lead to decreased myocardial velocity during the systolic phase after the procedure. After preload re- 
duction is accomplished, ventricular remodeling may occur slowly and lead to decreased myocardial velocity during the diastolic phase at follow-up. Previous studies have reported similar results; that is, changes in myocardial velocity after closure of a left-to-right shunt using a transcatheter device, which might have a volume reduction effect instead of inducing a real change in the myocardial velocity. Regional wall motion has been investigated in the right and left ventricles after device closure of atrial septal defects with chronic right ventricular volume overload. ${ }^{17)}$ In such cases, conventional function parameters showed no definite changes, but the systolic and diastolic myocardial velocities decreased significantly. However, a new marker, isovolumic acceleration (IVA), was found to provide a more independent measurement of ventricular loading conditions than did myocardial velocities. ${ }^{18)}$ Despite significant changes in the systolic and diastolic myocardial velocity, the IVA remained stable. The changes in myocardial velocity were considered to be a response to the modified ventricular loading conditions.

\section{Study limitations}

The major limitation of this study was its retrospective nature. In addition, variation in the physicians' measurements of echocardiographic parameters was not considered. Not all echocardiographic parameters were studied. For instance, IVA was not included.

\section{Conclusion}

This study demonstrated that, in patients undergoing percutaneous transcatheter PDA closure, larger amounts of pre-closure left-to-right shunting and higher pulmonary artery pressure were associated with increased likelihood of post-closure FS $<29 \%$. The initial Qp/Qs and $\mathrm{Pp} / \mathrm{Ps}$ ratios measured in the catheterization suite may be good predictors of LV dysfunction. In patients with high $\mathrm{Qp} / \mathrm{Qs}$ and $\mathrm{Pp} / \mathrm{Ps}$ ratios, serial assessment of ventricular function is recommended at follow-up echocardiography after PDA occlusion, as is evaluation for residual shunting.

\section{Acknowledgments}

This work was supported by the Korea Research Foundation Grant funded by the Korean Government (MOEHRD) (KRF-2007-531E00039).

\section{REFERENCES}

1) Takahashi Y, Harada K, Ishida A, Tamura M, Tanaka T, Takada G. Changes in left ventricular volume and systolic function before and after the closure of ductus arteriosus in full-term infants. Early Hum Dev 1996;44:77-85.
2) Galal MO, Amin M, Hussein A, Kouatli A, Al-Ata J, Jamjoom A. Left ventricular dysfunction after closure of large patent ductus arteriosus. Asian Cardiovasc Thorac Ann 2005;13:24-9.

3) Masura J, Tittel P, Gavora P, Podnar T. Long-term outcome of transcatheter patent ductus arteriosus closure using Amplatzer duct occluders. Am Heart J 2006;151:755, e7-755, e10.

4) Thanopoulos BD, Hakim FA, Hiari A, et al. Further experience with transcatheter closure of the patent ductus arteriosus using the Amplatzer duct occluder. J Am Coll Cardiol 2000;35:1016-21.

5) Faella HJ, Hijazi ZM. Closure of the patent ductus arteriosus with the amplatzer PDA device: immediate results of the international clinical trial. Catheter Cardiovasc Interv 2000;51:50-4.

6) Bilkis AA, Alwi M, Hasri S, et al. The Amplatzer duct occluder: experience in 209 patients. J Am Coll Cardiol 2001;37:258-61.

7) Hofbeck M, Bartolomaeus G, Buheitel G, et al. Safety and efficacy of interventional occlusion of patent ductus arteriosus with detachable coils: a multicentre experience. Eur J Pediatr 2000; 159:331-7.

8) Alwi M, Kang LM, Samion H, Latiff HA, Kandavel G, Zambahari R. Transcatheter occlusion of native persistent ductus arteriosus using conventional Gianturco coils. Am J Cardiol 1997;79: 1430-2.

9) Her K, Lee JW, Shin HK, Won YS. Video-assisted thoracoscopic surgery for patent ductus arteriosus. Korean Circ J 2004;34:97882.

10) Galal MO, Arfi MA, Nicole S, Payot M, Hussain A, Qureshi S. Left ventricular systolic dysfunction after transcatheter closure of a large patent ductus arteriosus. J Coll Physicians Surg Pak 2005; 15:723-5.

11) Steinherz LJ, Graham T, Hurwitz R, et al. Guidelines for cardiac monitoring of children during and after anthracycline therapy. Pediatrics 1992;89:942-9.

12) Porstmann W, Wierny L, Warnke H. The closure of the patent ductus arteriosus without thoracotomy (preliminary report). Thoraxchir Vask Chir 1967;15:199-203.

13) Masura J, Walsh KP, Thanopoulous B, et al. Catheter closure of moderate- to large-sized patent ductus arteriosus using the new Amplatzer duct occluder: immediate and short-term results. J Am Coll Cardiol 1998;31:878-82.

14) Park SS, Kim JH, Kwon MJ, Jung SW, Kim JK, Lee JH. Postnatal change of left ventricular contractility and volume in healthy term infants with ductus arteriosus. Korean Circ J 2000;30:1423-9.

15) Eerola A, Jokinen E, Boldt T, Pihkala J. The influence of percutaneous closure of patent ductus arteriosus on left ventricular size and function: a prospective study using two- and three-dimensional echocardiography and measurements of serum natriuretic peptides. J Am Coll Cardiol 2006;47:1060-6.

16) Jeong YH, Yun TJ, Song JM, et al. Left ventricular remodeling and change of systolic function after closure of patent ductus arteriosus in adults: device and surgical closure. Am Heart J 2007; 154:436-40.

17) Pauliks LB, Chan KC, Chang D, et al. Regional myocardial velocities and isovolumic contraction acceleration before and after device closure of atrial septal defects: a color tissue Doppler study. Am Heart J 2005;150:294-301.

18) Dalsgaard M, Snyder EM, Kjaergaard J, Johnson BD, Hassager C, Oh JK. Isovolumic acceleration measured by tissue Doppler echocardiography is preload independent in healthy subjects. Echocardiography 2007;24:572-9. 